\title{
THERMOMECHANICAL EFFECTS DURING DIRECT CHILL AND ELECTROMAGNETIC CASTING OF ALUMINUM ALLOYS PART II : NUMERICAL SIMULATION
}

\author{
J.-M. Drezet , M. Rappaz \\ Laboratoire de Métallurgie Physique, Ecole Polytechnique Fédérale de \\ Lausanne, $\mathrm{CH}$-1015 Lausanne, Switzerland \\ Y. Krähenbühl \\ Alusuisse Lonza Services Ltd. \\ Research and development \\ CH-3965 Chippis, Switzerland
}

\begin{abstract}
The prediction of the ingot deformation during direct chill (DC) and electromagnetic (EM) casting of aluminum alloy slabs would allow the optimization of the mold/inductor shape capable of producing flat ingots. The transient thermomechanical model presented here predicts the deformation and the temperature field evolution during DC/EM casting. Deformation in the solid is assumed to obey a viscoplastic law. The model is validated on the basis of the measurements presented in part I. It enables to predict the influence of casting parameters on butt curl and swell, rolling faces pull-in and residual stress state for DC and EM-cast ingots.
\end{abstract}

\section{Introduction}

Direct chill casting (DCC) and electromagnetic casting (EMC) proceed in two main stages (1): the start phase, during which the temperature field, the solidification front, the ingot shape and the liquid metalair interface (meniscus) change with time (nonstationary phase) and the pseudo-stationary phase. The deformations associated with the start phase, i.e. butt curl and butt swell, are due to the appearance of large thermal stresses which are induced when the butt enters the direct-chill zone. This non-stationary phase is critical because cracks can be initiated and the liquid metal must be confined at any time, especially during EMC, even in the presence of fast changing conditions at the start of the drop (2).

After about one meter of casting, a nearly steadystate regime is established for the thermal field. Under pseudo steady-state conditions, the solidified shell contracts inwards (i.e. towards the liquid pool) but not uniformly throughout the ingot cross-section.
If a rectangular mold/inductor is employed, the lateral faces of the resulting ingot are concave ("bone shape"). To compensate for the larger pull-in measured at the center of the rolling faces, the sides of the mold are designed with a convex shape, usually with three linear segments. Figure 1 shows a schematic top view of a typical mold shape used to produce flat rolling ingots. At the present time, the mold geometry is designed by a trial-and-error method.

Quantitative predictions of the deformations which occur during both the start and stationary phases would help to optimize the casting conditions and mold design capable of producing flat ingots.

\section{Steady-state inward pull-in of the rolling faces}

The deformation of the ingot cross-section during the stationary regime of semi-continuous DC-casting and the mold design required to produce flat sheet ingots have been studied by Weaver $(3,4)$. Without giving a physically-based explanation of this deformation, Weaver proposed an empirical model based on casting trials to predict the cross-section changes of DCC ingots. Assuming that the heat extraction was radial (i.e., parabolic shape of the isotherms in the metal under the liquid pool), Weaver derived the relationship:

$$
M O(x)=K_{1} \cdot T+K_{2}(x) \cdot T^{2} \cdot V
$$

where $M O$ is the mold opening as defined in figure $1, \mathrm{x}$ is the position on the longitudinal axis, $T$ is the desired ingot thickness and $V$ is the casting speed. The two alloydependent constants, $K_{1}$ and $K_{2}$, have to be determined from experiments. $K_{1}$ corresponds to a uniform thermal contraction and $K_{2}$ is a pull-in function which depends on $\mathrm{x}$. Weaver also showed that the mold design required to 
produce flat ingots changed from a two-straight lines shape, to an almost sinusoidal shape and eventually to a three-straight lines shape when the aspect ratio of the ingot (width over thickness ratio) was increased (4).

\section{Thermomechanical models}

Modeling of thermal stresses during DC casting has been performed by Fjaer and Mo for aluminum billets (5). Theses authors developed the finite element model Alspen for the calculations of the thermallyinduced strains and stresses that develop during the casting process. This model assumes the metal to be an isotropic elasto-viscoplastic material with strongly temperature-dependent properties (6). Mariaux et al. (7) developed a model which couples thermal aspects with mechanical effects. They computed butt curl during the EMC start phase. Hannart et al. (8) recently calculated the thermal stresses in DC casting of aluminum slabs with special emphasis on the induced stresses, which play a major role in the initiation of cracks. Their threedimensional transient thermomechanical analysis allows the prediction of butt curl and stress building during the start phase of DC casting.

However, in all these models, little attention was paid to the inward pull-in of the rolling faces which occurs in the pseudo steady-state regime of both DCC and EMC processes. This is precisely the goal of the present contribution. The model briefly described in section 2 will be validated in section 3 by comparison with the measurements presented in Part I.

\section{The model}

The aim of the present model is to assess precisely the influence of the process parameters (casting speed, mold shape, cooling conditions, etc.) and of the alloy composition on the ingot thermomechanical behavior. The model accounts for the coupled thermal and mechanical effects: the temperature gradients induce thermal stresses which deform the ingot and these deformations modify in counter part the heat extracted through the bottom block or the contact with the mold. In order to predict the inward rolling faces pullin that occurs in the pseudo steady-state regime, transient thermomechanical computations are carried out on a variable domain from the start phase to the pseudo steady-state regime.

\section{Computation domain}

Figure 2 shows the computation domain used for the study of the contraction of the rolling faces. Since three-dimensional transient computations are very intensive, the present investigation was limited in a first step to two dimensions. The computation domain shown on figure 2 corresponds to one of the plane of symmetry of the slab. Since this mid-plane section is perpendicular to the rolling faces, the heat flow is assumed to be twodimensional and mechanical aspects are reduced to a plane-deformation problem. The boundary conditions that are applied to DCC, are summarized in figure 3.

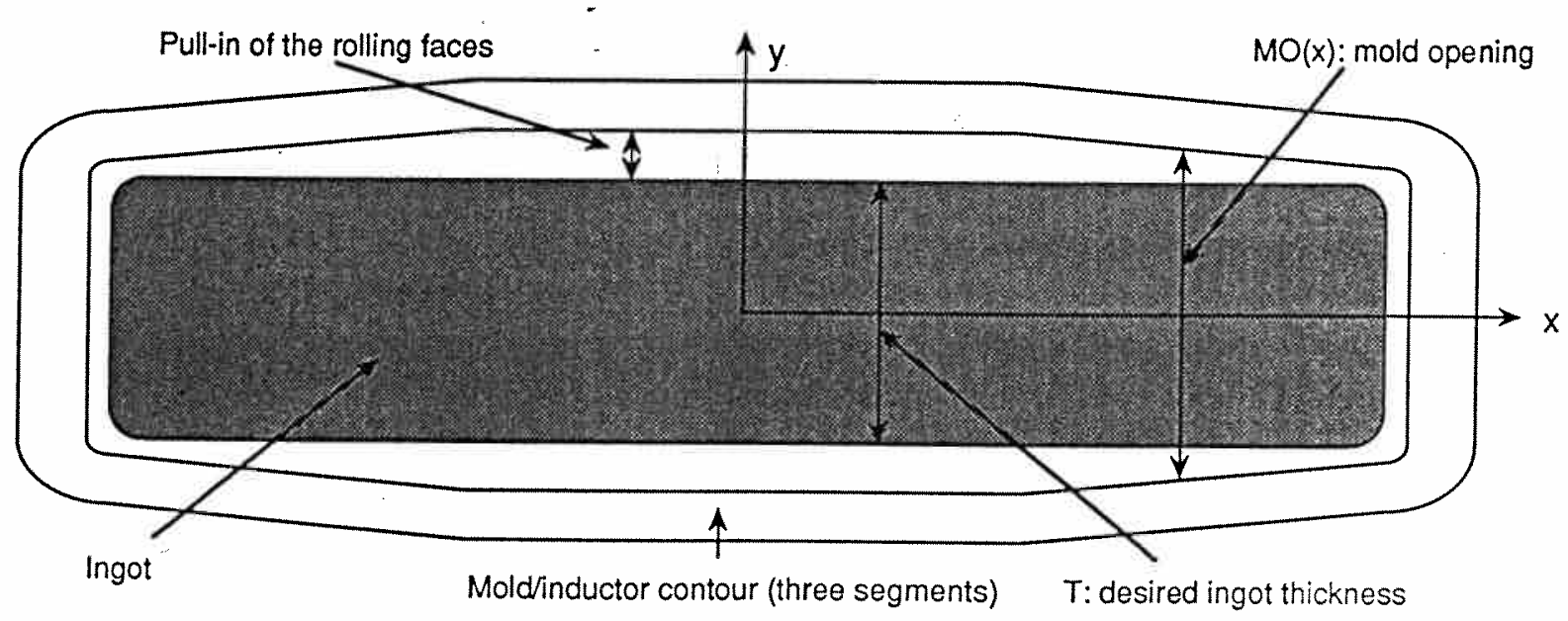

Figure 1: Schematic top view of the mod/inductor shape required to produce flat rolling sheet ingots. 


\section{Thermal aspects}

The thermal evolution is predicted by solving the heat conduction equation:

$$
\rho c^{e} p \partial T / \partial t+\operatorname{div}(k \cdot \operatorname{grad} T)=0
$$

where $\mathrm{k}$ is the thermal conductivity, $\rho$ is the density and $\mathrm{T}$ is the temperature field. $c^{e} p$ is the equivalent specific heat which accounts for the latent heat released during the liquid-solid phase transition. The thermal problem is solved in a two-dimensional domain which includes the ingot and the bottom block (see figure 3 ). The boundary conditions that are specified at the surfaces S1 to S6 are as follows:

- $\mathrm{S} 1$ is adiabatic for symmetry reason;

- the thermal fluxes through S2 and S3 are assumed to obey a Cauchy type law with a heat transfer coefficient

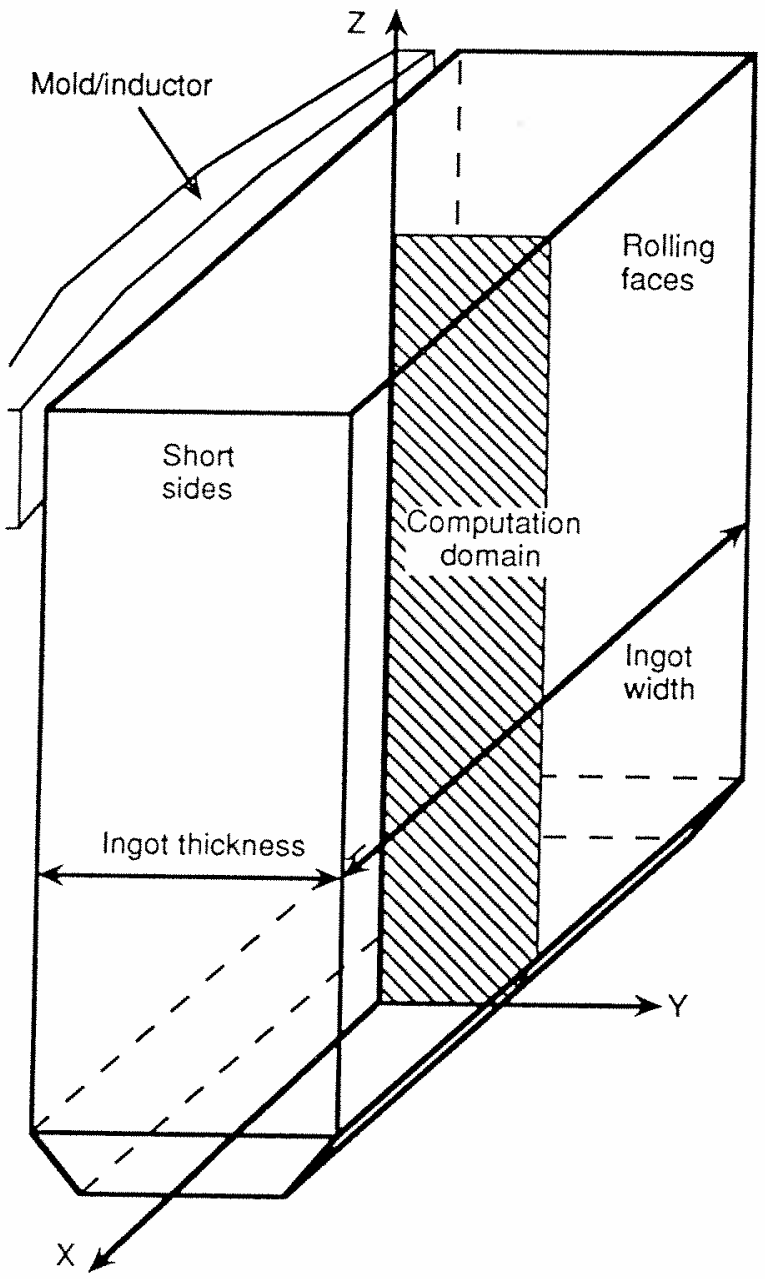

Figure 2: Computation domain. of $5000 \mathrm{~W} / \mathrm{m}^{2} \mathrm{~K}$ and a sink temperature of $10^{\circ} \mathrm{C}$;

at the ingot-bottom block interface $\mathrm{S} 4$, the corresponding boundary condition is $\mathrm{k}_{\mathrm{i}} \partial \mathrm{T} / \partial \mathrm{n}=\mathrm{kb}_{\mathrm{b}} \partial \mathrm{T} / \partial \mathrm{n}$ $=-h_{\text {gap }}\left(\mathrm{T}_{\mathrm{i}}-\mathrm{T}_{\mathrm{b}}\right)$ where $\mathrm{T}_{\mathrm{i}}$ and $\mathrm{T}_{\mathrm{b}}$ and $\mathrm{k}_{\mathrm{i}}$ and $\mathrm{k}_{\mathrm{b}}$ are the ingot and the bottom block surface temperatures and conductivities respectively; $\mathrm{n}$ is the normal to the boundary pointing outside the ingot. The contact quality and the gap opening calculated from the mechanical

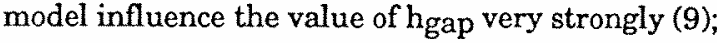

- S5 is the lateral surface of the ingot where the cooling is represented by a heat transfer coefficient depending on the position and on the process (DCC or EMC):

- at the top of the mold where the alloy is in direct contact with the mold (DCC), the prescribed heat transfer coefficient is set to a given value until the ingot temperature is lower than the coherency temperature of the alloy corresponding to the temperature at which air gap forms (see part I); this primary cooling is absent for EM casting;

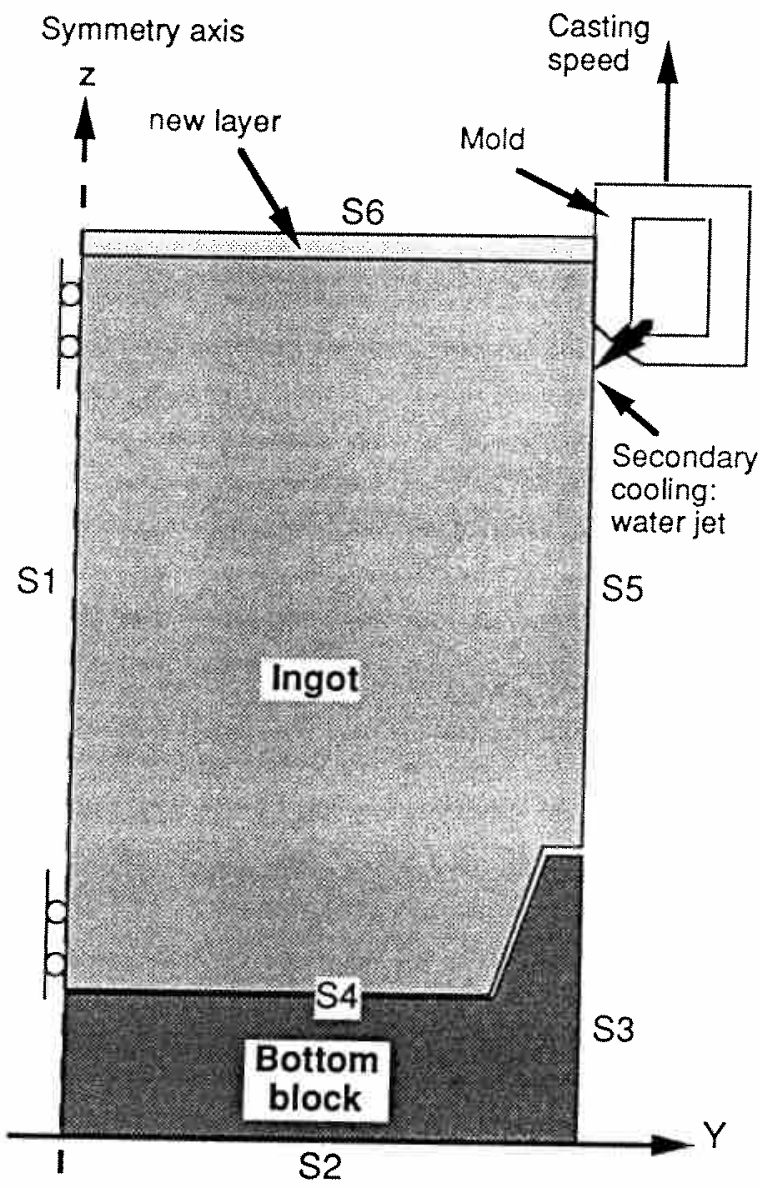

Figure 3: Schematic representation of the calculation domain with the its boundaries. 
- after the air gap has formed in DCC, the surface of the ingot is assumed to be almost adiabatic until the point of impact of the water cooling;

- for the remaining part of S5, the ingot is cooled down by water (jet or spray). The corresponding heat transfer coefficient depends on the ingot surface temperature and on the water flow rate; it was estimated using inverse modeling form the temperature histories recorded during casting (see Part I);

- 56 is the moving boundary of the domain. The incoming flow of liquid metal is modeled by the activation of successive layers of the enmeshment. The temperature of the new volume element is set to that of the incoming metal and the rate at which new layers are introduced corresponds to the casting speed.

\section{Mechanical aspects}

Equations

With the assumption of instantaneous equilibrium, the variation of the internal stress tensor, $\delta \alpha$, during a time interval, $\delta t$, is obtained in terms of the external force field variation, $\delta$, by solving the following incremental equation:

$$
d i v \delta \sigma+\delta f=0
$$

The incremental deformation tensor $\delta \&$ is assumed to be a superposition of elastic, thermal and viscoplastic components $\delta \varepsilon_{e}, \delta \varepsilon_{\text {th }}$ and $\delta \varepsilon_{\mathrm{vp}}(10)$ :

$$
\delta \varepsilon=\delta \varepsilon_{e}+\delta \varepsilon t h+\delta \varepsilon v p
$$

A two dimensional Cartesian model with planar deformation, i.e. $\delta \varepsilon_{x x}=\delta \varepsilon_{x y}=\delta \varepsilon_{x z}=0$ has been used. The elastic deformations are related to the internal stresses by Hooke's law $\delta \sigma=(D) \cdot \delta \varepsilon_{e}$, where (D) is the stiffness matrix defined in terms of the Young and Poisson modules, $E$ and $v$. The thermal deformations are related to the local temperature variation, $\delta \mathrm{T}$, through the equation $\delta \varepsilon_{i j}=\alpha \cdot \delta \mathrm{T} \cdot \delta_{\mathrm{ij}}$ where $\alpha$ is the linear expansion coefficient and $\delta_{\mathrm{ij}}$ is the Kronecker symbol. The model for viscoplastic deformations is presented in more details in a separate section.

Boundary conditions

The bottom block is assumed to deform elastically; the central part of S2 is mechanically fixed, whereas the remaining part is free. At each point $P$ of the boundary $\mathrm{S} 4$, the contact between the ingot and the bottom block is characterized by a separation distance $\mathrm{d}$; if $\mathrm{d}$ is larger than $100 \mu \mathrm{m}$, the gap is considered open and the heat transfer is set to its lower value. Friction is neglected and a no-penetration condition is imposed at this interface. The points located on the boundaries S5 and $\mathrm{S} 6$ are free to move, whereas these on $\mathrm{S} 1$ are free to slide along the z-axis.

\section{Thermoelastic behavior}

The computation domain includes the liquid metal, the mushy zone, the solid and the bottom block. The liquid metal is assumed to be incompressible and fluid motion is neglected. The temperature dependence of the Young and Poisson modules and of the dilatation coefficient are taken into account $(11,12)$. In the mushy zone, these parameters are linearly interpolated between their values in the liquid and solid. The contribution of the metal contraction during solidification is neglected owing to the constant liquid feeding within the mushy zone to compensate for the density variation (13).

Viscoplastic behavior

The time dependence of the viscoplastic deformations usually shows three distinct stages: a primary creep phase during which the creep rate rapidly decreases, a secondary phase with a nearly constant creep rate and finally the stage just before rupture (14). At high temperature, the primary stage is neglected and the viscoplastic behavior of the solid is assumed to be given by the Garafalo law (15):

$$
d \varepsilon_{u p} / d t=A \cdot\left(\sinh \left(\sigma_{v m} / \sigma\right)\right)^{n} \cdot \exp (-Q / R T)
$$

In the mushy zone, the Norton law is used:

$$
d \varepsilon_{v p} / d t=A^{\prime} \cdot\left(\sigma_{v m} / \sigma^{\prime}\right) n^{\prime} \cdot \exp \left(\cdot Q^{\prime} / R T\right)
$$

$\sigma_{\mathrm{Vm}}$ is the equivalent stress of Von Mises and the incremental viscoplastic deformation tensor is given by:

$$
\delta \varepsilon_{u p}=3 / 2 \cdot\left(d \varepsilon_{v p} / d t\right) \cdot \sigma_{d} / \sigma_{v m} \cdot \delta t
$$

where the deviatoric stress tensor is defined by $\sigma_{d}=\Omega$. $\operatorname{tr}(\sigma) \cdot \mathrm{Id} / 3$. 
The coefficients $A$ and $A^{\prime}$, the stresses $\sigma_{0}$ and $\sigma_{0}$, the creep activation energies $Q$ and $Q^{\prime}$ and the exponents $n$ and $n$ ' have been fitted using creep measurements (16) and indentation tests in the semi-solid state (17) for the alloys AA1201 and AA3104. The values for pure aluminum were taken from the work of Wong et al. (18).

\section{Numerical procedure}

The finite element program ABAQUS has been employed to perform the numerical computations. It allows the user to specify complex viscoplastic behavior of materials and flexible boundary conditions through user-subroutines (19). Successive layers of elements can be added to simulate the evolution of the casting shape. The fully coupled heat transfer/stress analysis is performed using 4 nodes quadrilateral elements, bilinear in displacement and temperature. The non-linearities introduced by the material properties (creep and latent heat) and by the contact have been treated by a Newton-Raphson integration scheme: at each time step, iterations, with an updating of the material characteristics, are made until both thermal and mechanical equilibrium are reached within the tolerances set by the user. An implicit integration scheme was used and the typical time step is of the order of $0.1 \mathrm{sec}$. The computation time was about 24 hours on a HP-735 workstation for a domain including 2000 nodes and for about 1.5 hour of casting.

\section{$\underline{\text { Results }}$}

The results of the temperature and deformation field computation are presented in this section for two alloys, AA1201 and AA3104, and for pure aluminum. The calculated pull-in of the rolling faces are compared with the measurements presented in Part I. The

\begin{tabular}{|c|c|c|}
\hline & Case 1 & Case 2 \\
\hline $\begin{array}{c}\text { casting process } \\
\text { nominal ingot } \\
\text { cross section }\left(\mathrm{mm}^{2}\right)\end{array}$ & DC-casting & EM-casting \\
\hline mold opening in the center & $550 \mathrm{~mm}$ & $546 \mathrm{~mm}$ \\
\hline alloy & $\mathrm{AA} 1201$ & $\mathrm{AA} 3104$ \\
\hline casting speed & $70 \mathrm{~mm} / \mathrm{min}$ & $65 \mathrm{~mm} / \mathrm{min}$ \\
\hline initial melt temperature & $670^{\circ} \mathrm{C}$ & $670^{\circ} \mathrm{C}$ \\
\hline cooling time after casting & 1 hour & 1 hour \\
\hline
\end{tabular}

Table I: Casting conditions of case 1 and 2. calculations were performed until a pseudo steady-state was reached, from both a thermal and a mechanical point of view. In order to obtain such conditions, it was found that a cast length of at least $1.5 \mathrm{~m}$ had to be modeled. For precaution, the results presented here are for 2.2 meter final length castings.

\section{Comparison with experiments}

Two calculations were first carried out for comparison with the measured pull-in (see Part I). The casting conditions are given in Table 1 . In the first case (DCC), the liquid level was $65 \mathrm{~mm}$ below the top of the mold and in EMC (case 2), the distance between the meniscus and the electromagnetic screen was $6 \mathrm{~mm}$. Figure 4 shows the temperature profile of the ingot just at the end of casting, i.e., as the metal feeding is stopped, and a map of the $y$-displacement (see figure 3) after
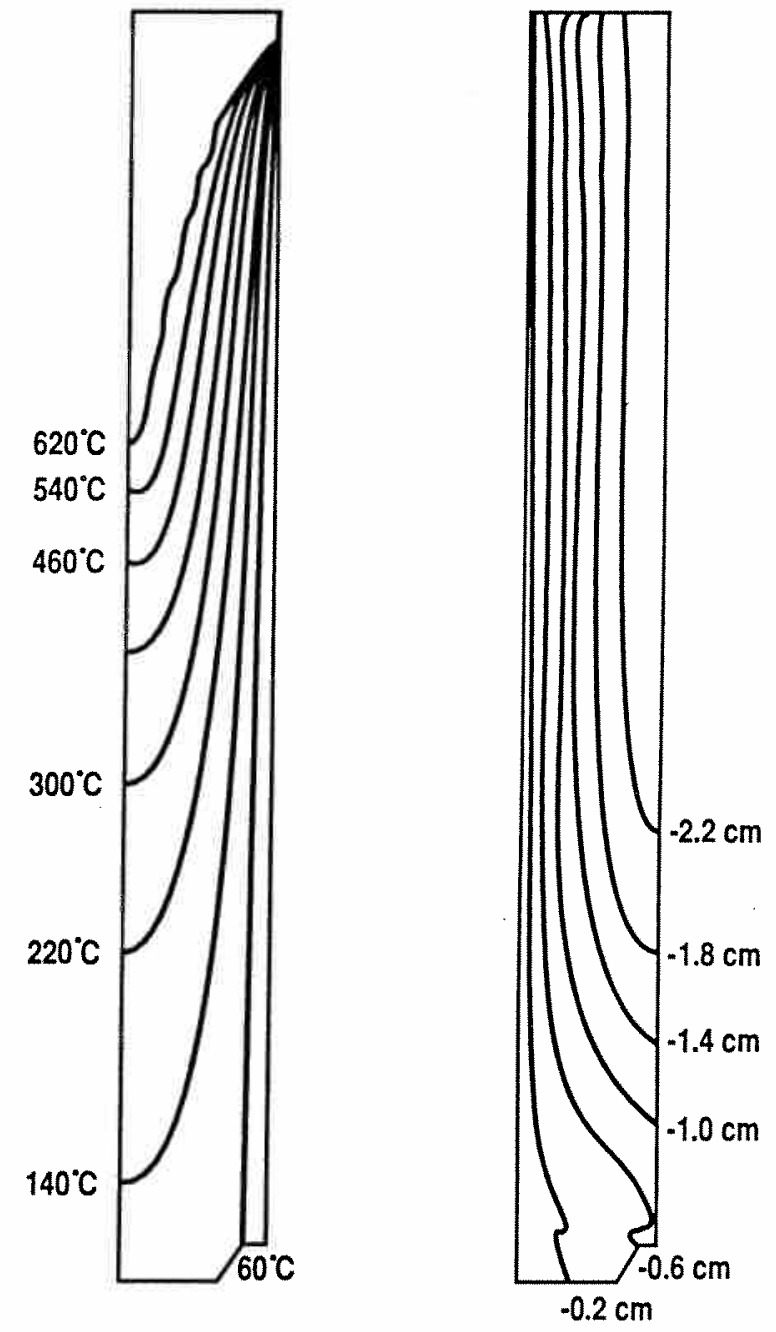

Figure 4: Temperature field (left) at the end of casting and $y$-displacement map (right) after cooling (case 1). 
complete cooling, which clearly demonstrates that a stationary regime had been reached. The surface temperature and contraction profiles are reported in figure 5 at the end of casting and after cooling. The calculated and measured ingot profiles along the $z$ direction after cooling are compared for both DCC (figure 6) and EMC (figure 7). As can be seen, the agreement is quite good, considering that the model is twodimensional.

\section{Influence of the casting speed}

Figure 8 shows the pull-in profile of a DC-cast AA3104 ingot just at the end of casting for three casting speeds. Figure 9 presents the same profile after complete cooling. Finally, Figs. 10 and 11 compare the calculated and measured rolling face pull-in as a function of the casting drop for the two alloys AA3104 and AA1201, respectively. These values correspond to the steady-state pull-in obtained after cooling. The measured and calculated sump depth are reported in the same diagram. Again, a good agreement is found.
Influence of the alloy composition

Figure 12 compares the sump depth and the steady-state rolling faces pull-in calculated after complete cooling as a function of the solidification range. As can be seen, pure aluminum deforms much less than the alloys AA1201 and AA3104.

\section{Influence of the mold opening}

The influence of the mold opening, and consequently of the ingot thickness, on the steady-state rolling faces pull-in and sump depth is shown in figure 13 for the alloy AA1201 DC-cast at $70 \mathrm{~mm} / \mathrm{min}$. The ingot width has been kept constant $(1860 \mathrm{~mm})$.

\section{Residual stresses}

The residual stresses $\sigma_{y y}, \sigma_{z z}$ and $\sigma_{y z}$ calculated for the DC-cast ingot after complete cooling are shown in figure 14. The ingot surface is in compression $\left(\sigma_{y y}, \sigma_{z z}\right)$ whereas the heart is in tension; this is in accordance

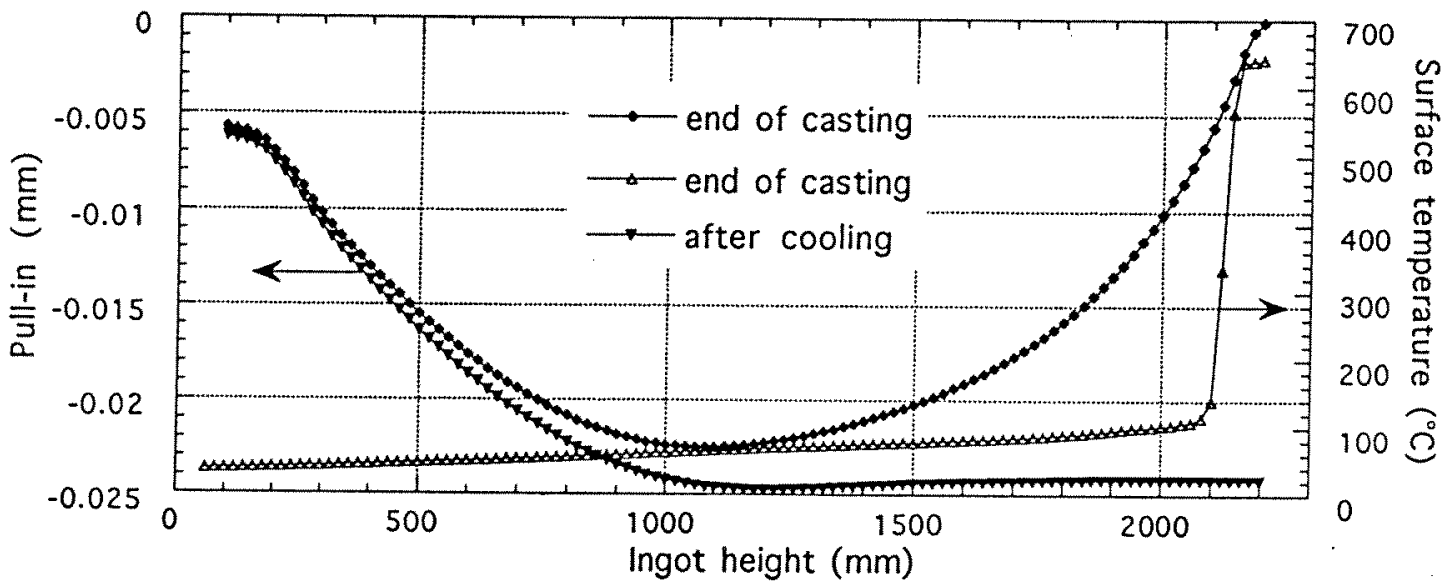

Figure 5: Ingot surface temperature and rolling face pull-in (DCC,case 1).

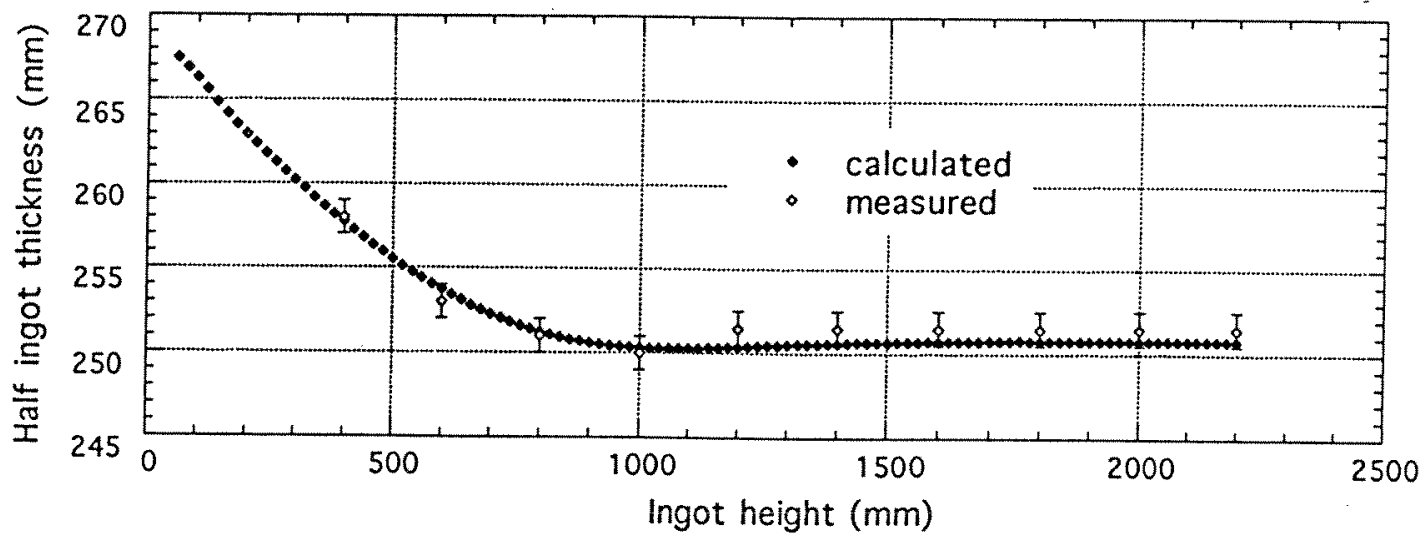

Figure 6: Comparison between measured and calculated ingot profile for DCC (case 1). 
with the work of Fjaer and Mo $(5,6)$. The shear stress $\sigma_{\mathrm{yz}}$ is almost zero in the center part of the ingot. Such results can be very useful to optimize the temperature and the holding time necessary to relieve the thermal stresses before rolling.

\section{Discussion}

The agreement between calculated and measured ingot profiles is very satisfactory for both DCC (figure 6) and EMC (figure 7). As shown in Part I, most of the ingot contraction occurs below the mold/inductor as a result of large thermal gradient induced upon cooling. In DCC, the cooling can be subdivided in two steps, the first one being associated with the contact with the mold and the second one arising when the water jet strikes the surface. Although the measured pull-in clearly reveals these two stages (see Part I), the contraction of the ingot at the exit of the mold only represents $9 \%$ of the total pull-in after cooling. In EMC, there is only one cooling associates with the water spray and the contraction is continuous.

The calculation presented here also show that most of the contraction is induced by the cooling of the solid ingot. In figure 5 , it is seen that the last solid to have formed at the end of the casting has about the size fixed by the mold opening (i.e., contraction equals zero at

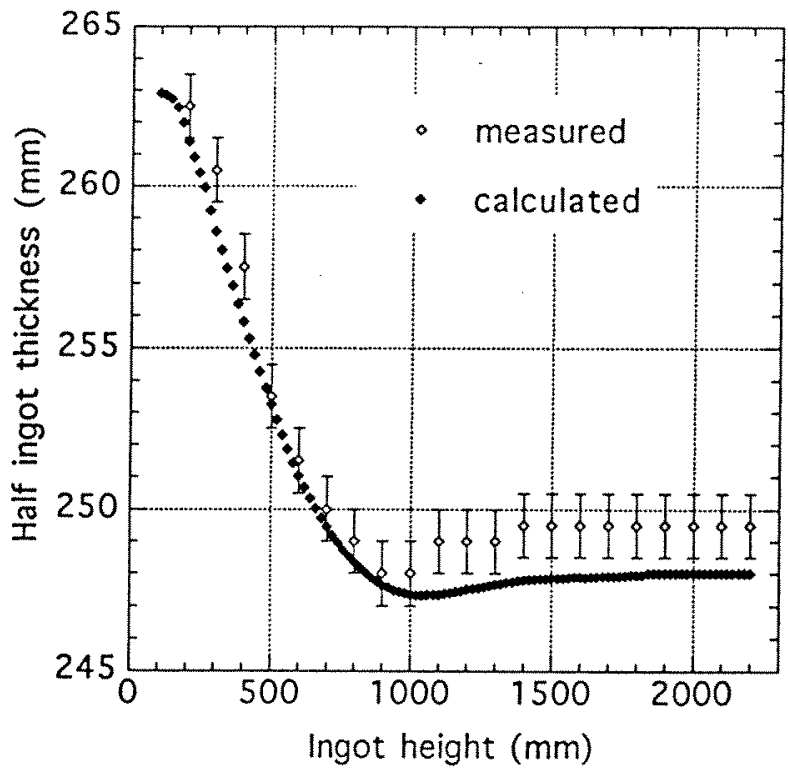

Figure 7: Comparison between measured and calculated ingot profile for EMC (case2).
2.2 meters when this part has just solidified). After cooling to room temperature, this part of the ingot experiences a $24 \mathrm{~mm}$ pull-in, i.e., about $10 \%$ contraction. As shown in Ref. 13, the ingot pull-in is affected by the shape of the liquid pool and by the horizontal component of the thermal gradient. The thermomechanical and thermophysical properties of the alloy being given, it is expected that the pull-in increases with the sump depth (see ref. 13). This trend is reflected in Figs. 9 to 11. As the casting speed is increased, the liquid pool becomes deeper and the thermal gradient near the cooling water

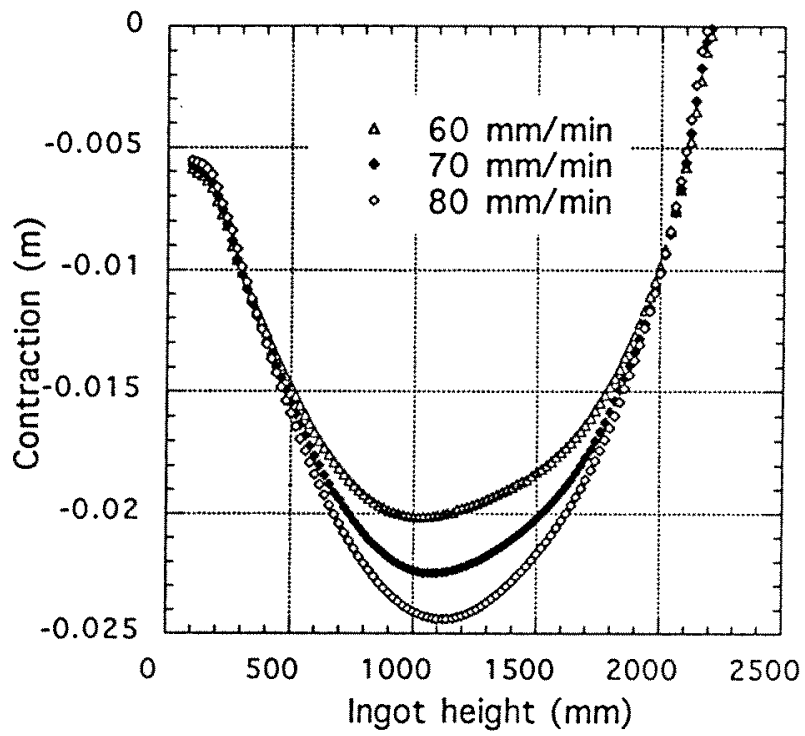

Figure 8: Effect of casting speed on the rolling face profile at the end of casting for a DC-cast AA3104 ingot.

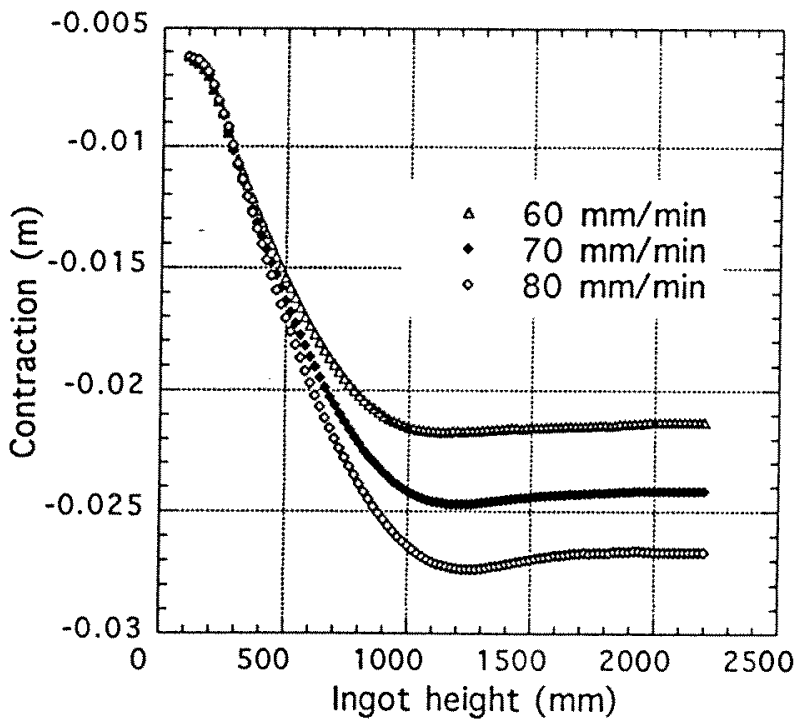

Figure 9: Effect of casting speed on the rolling face profile at the end of casting for a DC-cast AA3104 ingot. 
is more horizontal. As a result; the pull-in increases. The same trend is predicted when changing the mold opening: the ingot being thicker, the sump is deeper and the final pull-in is increased (figure 13).

The influence of the alloy composition is not as straightforward because both the thermomechanical and the thermophysical properties change. Under identical thermal conditions, the creep behavior of the ingot definitely changes the final pull-in as the material flows to accommodate the thermal stresses; for

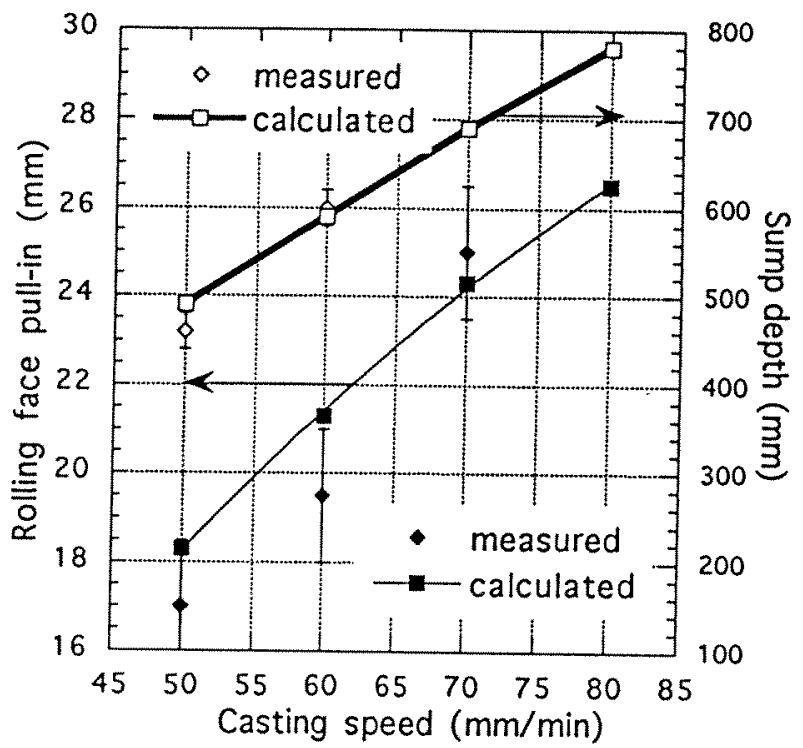

Figure 10: Comparison between calculated and measured sump depth and rolling face pull-in after cooling (AA3104, DCC, steady-state).

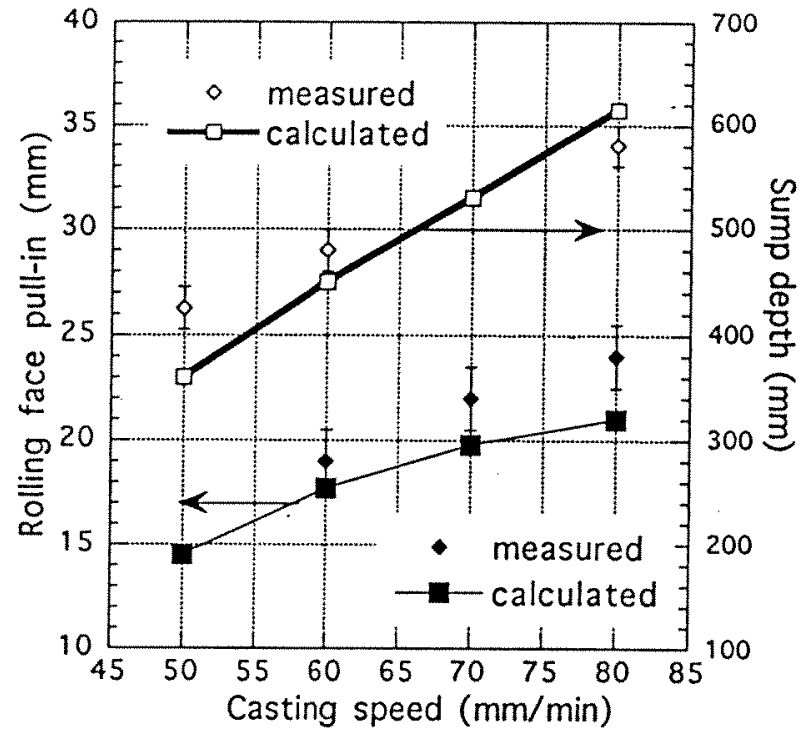

Figure 11: Comparison between calculated and measured sump depth and rolling face pull-in after cooling (AA1201, DCC, steady-state). example, pure aluminum and AA1201 (see figure 12) have comparable thermal conductivity, thus showing approximately the same sump depth, but contract differently due to their respective mechanical behavior. However, the thermophysical properties also play a role. The thermal conductivity is highest for pure aluminum, whereas the specific heat and latent heat are almost unaffected by the alloy composition. Therefore, the sump depth is smaller for pure aluminum in comparison with the alloy AA3104, and so will be the final ingot contraction as can be seen in figure 12 .

As can be seen, the final shape of DC and EMcast ingots is a result of several interplaying

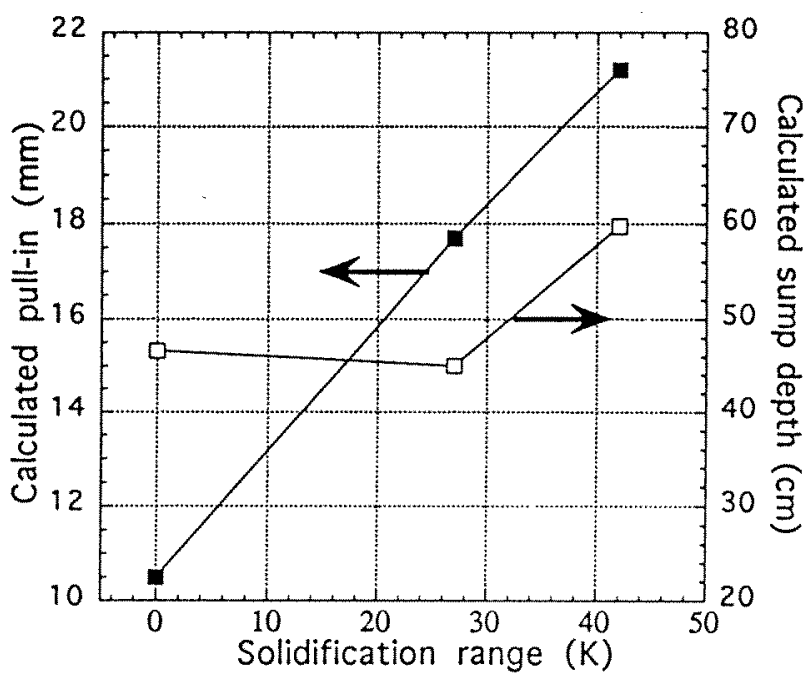

Figure 12: Calculated steady-state rolling face pull-in and sump depth as a function of the solidification range (DCC, $60 \mathrm{~mm} / \mathrm{min}$ ).

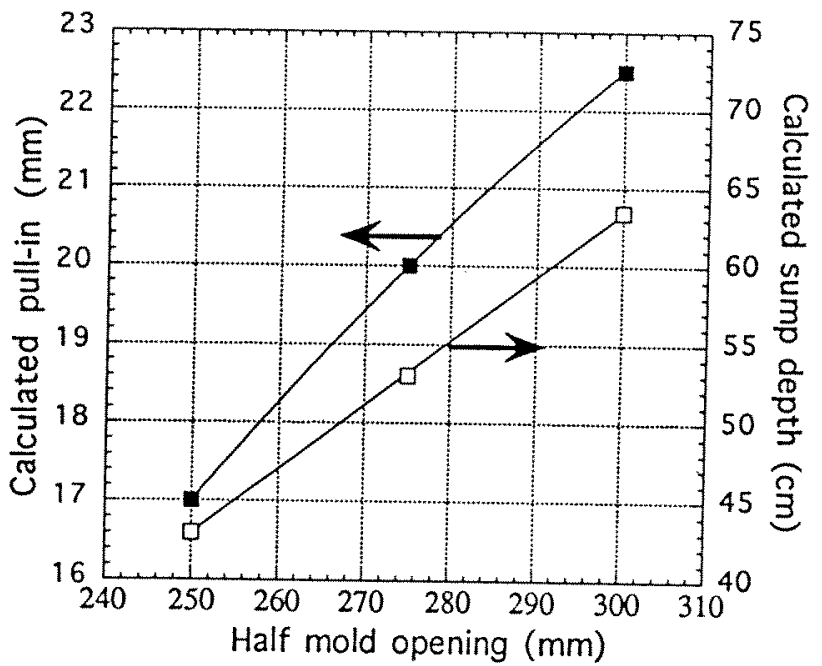

Figure 13: Rolling face pull-in and sump depth for the alloy AA1201 DC-cast at $70 \mathrm{~mm} / \mathrm{min}$ as a function of the mold opening. 
phenomena. However, the present study has clearly shown that most of the pull-in is associated with thermal stresses induced upon cooling of the solid and that the contraction at the exit of the mold/inductor is only a minor part of the overall pull-in. The horizontal component of the thermal gradient, which is reflected by the liquid pool profile, plays a key role in the deformation of the ingot. Good agreement can be found with the experiment providing the mechanical and thermophysical properties of the alloys are known up to the coherency temperature and the thermal boundary conditions are well characterized.

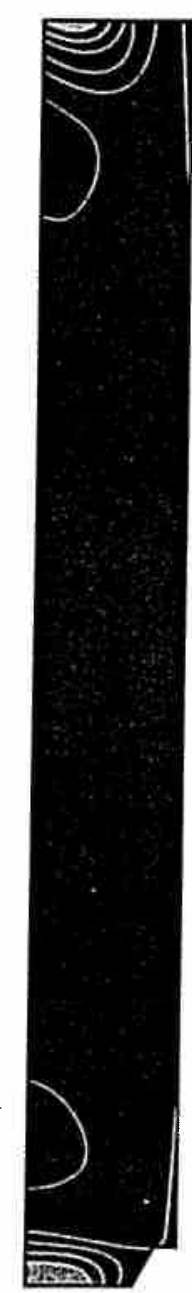

$\sigma 11\left[\mathrm{~N} / \mathrm{m}^{2}\right]$
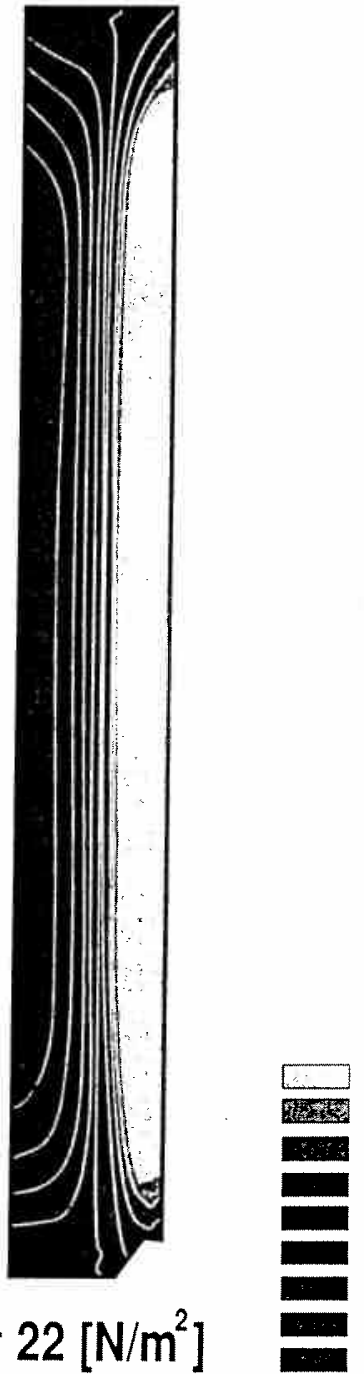

$-1.00 E+08$

$-7.50 E+07$

$-5.00 \mathrm{E}+07$

$-2.50 E+07$

$+0.00 E+00$

$+2.50 E+07$

$+5.00 E+07$

$+7.50 E+07$

$+1.00 E+08$

\section{Conclusion}

The two-dimensional thermomechanical model presented here has been validated using the experimental results of part I. Mechanical properties have been measured independently and thermal boundary conditions were adjusted to the measured cooling curves. The model is able to predict with a good accuracy the steady-state rolling faces pull-in as a function of the casting conditions (casting speed, ingot size) and alloy composition for both DCC and EMC. The assumption of plane deformation appears to be valid for

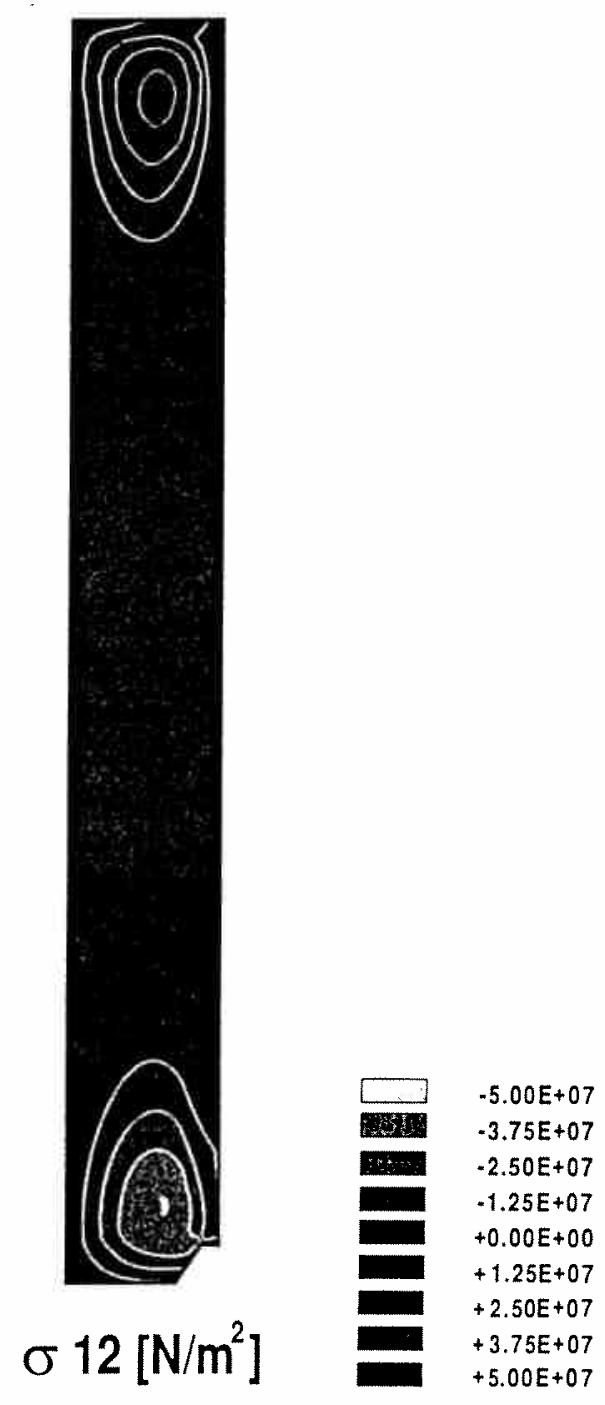

Figure 14: Residual stresses in the DC-cast AA1201 ingot ( $70 \mathrm{~mm} / \mathrm{min}$, case 1) after complete cooling $(1 \equiv \mathrm{y}, 2 \equiv z)$. 
the mid-plane section of the casting. However, an optimization of the mold bow would clearly require the use of a three-dimensional model, the short sides of the ingot having a much smaller contraction. The extension to three dimensions is limited only by the computing power of the workstations and the present contribution is already very valuable in assessing the key parameters controlling the rolling faces pull-in. For example, we clearly demonstrated that the large pull-in is not explained by the liquid-solid contraction (about 6\% in volume for aluminum) but by the cumulated and associated bending of the solid shell under the high thermal gradient (13). Three dimensional computations are under way using the same model, boundary conditions and thermophysical properties.

\section{Acknowledgment}

The financial support of the swiss Commission pour l'Encouragement de la Recherche Scientifique (CERS), under grant 2496.1, is greatly acknowledged.

\section{References}

1. E.F. Emley: Continuous casting of aluminum; International Metals Reviews, June 1976.

2. Y. Krähenbühl, R. Von Kaenel, B. Carrupt, J.C. Weber: Understanding the starting phase: a key factor to the success of EMC (electromagnetic casting); Light Metals, 1990, p. 893.

3. C.H. Weaver: An empirical model to explain crosssection changes of D.C. sheet ingot during casting, TMS, AIME, 1976 , p. 441.

4. C.H. Weaver: Designing sheet ingot molds to produce rectangular ingots of the desired thickness and width, Light Metals, 1991, p. 953.

5. H. Fjaer and A. Mo : Alspen-a mathematical model for thermal stresses in direct chill casting of aluminum billets, Met, Trans, vol. 21B, 1990, p. 1049.

6. H. Fjaer and A. Mo : Mathematical Modeling of Thermal Stresses during DC casting of Aluminum Billets, Light Metals, 1990, p. 945.

7. S. Mariaux, M. Rappaz, Y. Krähenbühl, M. Plata: Modeling of thermo-mechanical effects during the start-up phase of the electromagnetic casting process, Light Metals, 1992, p. 175.
8. B. Hannart, F. Cialti, R. V. Schalkwijk: Thermal stresses in DC casting of aluminum slabs: application of a finite element model, Light Metals, 1994, p. 879.

9. K. Ho and R. Pehlke : Metal-mold interfacial heat transfer, Met. Trans. , vol. 16B, 1985, p. 585.

10. L. Anand: Constitutive Equations for the RateDependent Deformation of Metals at Elevated Temperatures, Trans. ASME, vol. 104, 1982, p. 12.

11. L.F. Mondolfo : Aluminum Alloys: Structure and Properties, Butterworths, Boston, 1976.

12. Aluminum: Properties and Physical Metallurgy, edited by John E. Hatch, ASM, Metals Park, Ohio, 1983.

13. J.-M. Drezet, M. Rappaz, B. Carrupt and M. Plata : Experimental investigation of thermomechanical effects during direct chill and electromagnetic casting of aluminum alloys, to be published in Met. Trans.

14. Introduction to creep, R. W. Evans and B. Wilshire, The Institute of Materials.

15. F. Garafalo : An empirical relation defining the stress dependence of minimum creep rate in metals, Trans Met Soc AIME, vol. 227, 1963, p. 351.

16. J.M. Drezet and G. Eggeler: High apparent creep activation energies in mushy zone microstructures, Scripta Met et Mater, vol. 31, Sept. 1994, p. 757.

17. O. Branswyck, J. Collot, P. Vicente-Hernandez, A.M. Chaze and C. Levaillant: Investigation of semisolid state behavior of alloys aimed at process modeling, in Euromat 1991, Ed. J. W. Clyne (Inst. Metals, London 1992), p. 124-130.

18. W. A. Wong and J. J. Jonas: Aluminum extrusion as a thermally activated process, Trans. Met. Soc AIME, vol. 242, 1968, p. 2271.

19. Abaqus theoretical manual, K. Hibbit and J. Sorensen, Hibbit, Karlson and Sorensen, Inc., Providence, 1989. 